\title{
Addendum: On the ionization of a Keplerian binary system by periodic gravitational radiation [J. Math. Phys. 37, 3997-4016 (1996)]
}

C. Chicone

Department of Mathematics, University of Missouri, Columbia, Missouri 65211

B. Mashhoon

Department of Physics and Astronomy, University of Missouri, Columbia, Missouri 65211

D. G. Retzloff

Department of Chemical Engineering, University of Missouri, Columbia, Missouri 65211

(Received 9 September 1996; accepted for publication 17 September 1996)

[S0022-2488(97)02401-8]

In our paper, ${ }^{1}$ two equations have incorrect forms due to printing errors. The last equation on p. 4004 should read

$$
F^{L}(e, \ell, g)=U_{2 m}(e) \sin (2 g+2 m \ell)+\frac{\delta}{4}\left[(\alpha-\beta) U_{m}(e) \sin (2 g+m \ell)\right]
$$

and, similarly, the last equation on p. 4007 should read

$$
0=\left.\frac{d}{d \vartheta}\left[\Pi D \Delta(\Gamma(\vartheta), 0) \Upsilon^{\prime}(\vartheta)\right]\right|_{\vartheta=0}=\Pi D^{2} \Delta(\eta, 0)\left(\Gamma^{\prime}(0), \Upsilon^{\prime}(0)\right)+\Pi D \Delta(\eta, 0) \Upsilon^{\prime \prime}(0)
$$

Inclusion of radiative damping in our model due to gravitational radiation reaction is expected to lead to transient chaotic effects. ${ }^{2}$

${ }^{1}$ C. Chicone, B. Mashhoon, and D. G. Retzloff, “'On the ionization of a Keplerian binary system by periodic gravitational radiation," J. Math. Phys. 37, 3997-4016 (1996).

${ }^{2}$ C. Chicone, B. Mashhoon, and D. G. Retzloff, "Gravitational ionization: a chaotic net in the Kepler system," preprint (1996). 